Originally published as:

Böhmer, M.M., Walter, D., Krause, G., Müters, S., Gößwald, A., Wichmann, O.

Determinants of tetanus and seasonal influenza vaccine uptake in adults living in Germany (2011) Human Vaccines, 7 (12), pp. 1317-1325.

DOI: $10.4161 / h v .7 .12 .18130$

This is an author manuscript.

The definitive version is available at: http://www.landesbioscience.com/journals/hv/ 


\section{Determinants of tetanus and seasonal influenza vaccine uptake in adults living in Germany}

Merle M. Böhmer ${ }^{\mathrm{a}, \mathrm{d},{ }^{*}}$, Dietmar Walter ${ }^{\mathrm{a}, \mathrm{d}}$, Gérard Krause ${ }^{\mathrm{b}}$, Stephan Müters ${ }^{\mathrm{c}}$, Antje Gößwald ${ }^{\mathrm{c}}$, Ole Wichmann ${ }^{\mathrm{a}}$

${ }^{\text {a } I m m u n i z a t i o n ~ U n i t, ~ R o b e r t ~ K o c h ~ I n s t i t u t e, ~ B e r l i n, ~ G e r m a n y ~}$

${ }^{\mathrm{b}}$ Department for Infectious Disease Epidemiology, Robert Koch Institute, Berlin, Germany

${ }^{\mathrm{c}}$ Department of Epidemiology and Health Reporting, Robert Koch Institute, Berlin, Germany

${ }^{\mathrm{d}}$ Charité - University Medicine Berlin, Berlin, Germany

* Corresponding author:

Merle M. Böhmer

Immunization Unit,

Department for Infectious Disease Epidemiology,

Robert Koch Institute,

DGZ-Ring 1, 13086 Berlin, Germany

E-mail address: BoehmerM@,rki.de 


\section{Abstract}

The primary objective of this study was to assess determinants of vaccine uptake in adults living in Germany exemplified by one standard vaccination (tetanus) and one vaccination targeting specific risk-groups (seasonal influenza). Data from 21,262 telephone householdinterviews representative of the adult population in Germany were collected in 2009 and analyzed. A total $73.1 \%$ of the adult population had a sufficient tetanus vaccination status according to national recommendations (i.e. last tetanus shot $\leq 10$ years ago). Influenza vaccination coverage in the target population (i.e. persons $\geq 60$ years, chronically ill, healthcare workers) was $44.1 \%$. Persons who received professional vaccination advice within the past five years were more frequently vaccinated against tetanus and influenza than persons without $(\mathrm{p}<0.001)$. Private physicians were identified as the most important source for vaccination advice. Having a statutory health insurance, last physician contact $<1$ year ago, and living in the eastern part of Germany were independently associated with higher tetanus and influenza vaccine uptake. Low socio-economic status, two-sided migration background, and the feeling of being insufficiently informed on the benefits of vaccination were independently associated with low uptake of tetanus but not influenza vaccines. Our results show that tetanus vaccination coverage in the general adult population and influenza vaccination coverage in the target population are unsatisfactorily low in Germany. Since physicians' advice has a major impact on the vaccination decision, physician reminder systems could provide a method to increase vaccination coverage in adults. For tetanus, information activities should target population groups with an increased risk of being undervaccinated.

Keywords: vaccination, determinants, coverage, tetanus, influenza, Germany 


\section{Introduction}

With the success of vaccines in reducing the burden of various diseases, the threat posed by these diseases is less imminent and the benefits of vaccination programs are becoming less obvious to the public. To achieve high vaccination coverage in the target population for which a particular vaccination is recommended poses a challenge to public health authorities. Reliable data on the incidence and trends of vaccine-preventable diseases as well as on vaccination coverage in the target populations are needed to evaluate and, if necessary, to adjust vaccination programs by implementing appropriate strategies. In this context, the assessment of the impact of socio-economic, regional, informational and other health-relevant factors on vaccination coverage is important to identify potential barriers to high vaccine uptake.

In Germany, the Standing Committee on Vaccination (STIKO) develops and endorses vaccination recommendations on a national level. ${ }^{1}$ The sixteen federal states in Germany are responsible for the implementation of vaccination programs. Vaccines for adults are usually administered by general practitioners and in some companies also by occupational physicians. There are two different health insurance schemes in Germany: the statuary health insurance (SHI) and the private health insurance (PHI) scheme. Approximately $90 \%$ of the German population is covered by a SHI. ${ }^{2}$ STIKO-recommended vaccinations are free of charge for both, persons who are covered by SHI and by PHI. However, persons with PHI need to pay for the vaccine and its administration out-of-pocket, and reimbursement of these expenses takes place after the bills are submitted to the insurance company. In contrast, persons with SHI are not involved in any payments of the physician or the vaccines.

Tetanus and influenza are two diseases that can effectively be prevented by vaccination. In Germany, tetanus booster vaccination is recommended by STIKO to be given to all adults every ten years. ${ }^{1}$ Between 2001 and 2009, there were up to 32 reported cases of 
tetanus each year, which occurred mainly among elderly persons. ${ }^{3,4}$ However, the disease is notifiable only in 6 of the 16 federal states. Estimates of annual tetanus case numbers are therefore based on notification data from the respective states ${ }^{5}$ as well as on case reports ${ }^{4}$ and national hospital discharge statistics. ${ }^{3}$ Influenza vaccination is recommended for defined target groups who have either an increased risk for severe influenza disease (e.g. person with chronic underlying diseases, pregnant women, and persons $\geq 60$ years of age) or who are likely to transmit the virus to vulnerable groups (e.g. health care workers). ${ }^{1}$ Depending on the intensity of influenza-activity there are between zero and approximately 30,000 estimated excess deaths attributable to influenza in Germany each year. ${ }^{6}$

Germany has no central immunization registry. Data on vaccination coverage of children is systematically collected only at school entry when the children are between 5 and 6 years of age. ${ }^{7}$ Information on vaccination coverage in adolescents and adults have mainly been available from a few smaller telephone surveys ${ }^{8-11}$, from health insurance companies ${ }^{12}$ and household surveys (Microcensus 2003) ${ }^{13}$, and are largely insufficient.

With the implementation of the 'German Health Update' Survey (GEDA) in $2008 / 2009$ as a part of the national health monitoring, a tool is now available in Germany for the detailed assessment of vaccination coverage in all age-groups above 18 years. GEDA is a large, population-representative telephone-survey. Here we present results from GEDA 2009 with focus on one standard vaccination for adults (tetanus) and one vaccination targeting specific risk groups (influenza). While target-group specific influenza vaccination coverage based on GEDA 2009-data was subject of a previous publication ${ }^{14}$, this paper focuses in detail on factors influencing tetanus and seasonal influenza vaccine uptake. The objectives of this study were i) to assess the proportion of adults who were vaccinated against tetanus and seasonal influenza according to national recommendations, and ii) to identify factors 
potentially associated with vaccine uptake in the target populations thereby generating evidence for more targeted vaccination programmes. 


\section{Results}

Sample characteristics. In total, 21,262 persons were interviewed via telephone during the study period of GEDA 2009 (July 2008-June 2009). Response Rate 3 as defined by the American Association for Public Opinion Research (AAPOR) was 29.1\% and the cooperation rate at respondent level was $51.2 \% .{ }^{14,15}$ An overview of the study sample and the adult population in Germany (data of the German Federal Statistical Office, 2008) is given in Table 1 . The median age in the study sample was 48.0 years (range $18-100$ years). All data presented in the following sections are weighted data.

Tetanus vaccination coverage. Information on tetanus vaccination status (last tetanus shot $>$ or $\leq 10$ years ago) was available for 20,470 respondents $(96.3 \%$ of the study population). A total $73.1 \%$ (95\% confidence intervall (CI) $72.3-74.0)$ had a sufficient tetanus vaccination coverage according to the national recommendation (last tetanus shot $\leq 10$ years ago). Tetanus vaccination coverage decreased with age and was lowest in persons $\geq 70$ years (Figure 1). Among 20,840 respondents (98.0\% of the study population) with information available, $95.4 \%(95 \% \mathrm{CI} 95.0-95.9)$ stated to have received a tetanus shot at least once in their life.

The most frequently reported reason for receiving the last tetanus shot was that it was necessary due to the recommended immunization schedule (61.5\%; 95\%CI 60.6-62.4). A total $31.4 \%(95 \% \mathrm{CI} 30.6-32.3)$ reported to have received their last tetanus vaccination as part of an injury treatment, and $7.1 \%(95 \% \mathrm{CI} 6.7-7.5)$ of the interviewed persons received it during a pre-travel health consultation. Respondents belonging to the first group were significantly more frequently vaccinated as compared to those belonging to the second (tetanus vaccination coverage $82.9 \%$ vs. $68.6 \% ; p<0.001)$ or third group $(78.0 \% ; p<0.001)$. The majority of interviewed persons $(72.2 \%$; 95\%CI 71.3-73.0) reported to have received their last tetanus vaccination at their family doctor; $7.8 \%(95 \% \mathrm{CI} 7.3-8.3)$ received it at some other private 
physician, $14.2 \%(95 \% \mathrm{CI} 13.5-14.9)$ received it at a hospital emergency unit after injury, and $2.6(95 \%$ CI $2.3-2.9)$ received it at the local public health service.

Seasonal influenza vaccination coverage. Influenza vaccination status (vaccinated or unvaccinated for the last influenza season) was available for 21,190 interviewed persons (99.7\%). Of those 10,640 (50.2\%; 95\%CI: 48.9-50.7) belonged to at least one of the recommended target groups for influenza vaccination. Influenza vaccination coverage was $44.1 \%(95 \% \mathrm{CI} 42.7-45.4)$ in persons belonging to the target population and $17.0 \%(95 \% \mathrm{CI}$ 16.2-17.9) in the non-target population. In contrast to tetanus vaccination, influenza vaccination coverage increased with age and was highest in persons $\geq 70$ years (Figure 1). Among the different target groups, vaccination coverage was highest in persons $\geq 60$ years (56.3\%; 95\%CI 54.5-58.1) and lowest in healthcare workers (HCW) $(22.2 \%$; $95 \% \mathrm{CI} 19.7$ 25.0). The proportion vaccinated was $44.5 \%$ (95\%CI 42.8-46.1) among persons with underlying chronic diseases.

Factors associated with tetanus and seasonal influenza vaccine uptake. Results of univariate and multivariate analysis of factors potentially associated with tetanus and seasonal influenza vaccine uptake are presented in Table 2 and Table 3 respectively. Several factors were independently associated with both higher tetanus and influenza vaccine uptake including having a SHI, having received a professional vaccination advice within the past five years, physician contact in the last 12 months, and caring about personal health. Unlike influenza vaccination coverage, tetanus vaccination coverage decreased with increasing size of the city of residency and with age. Women were significantly less frequently vaccinated against tetanus but not influenza than men. Having a two-sided migration background decreased the odds of being vaccinated against tetanus by $40 \%$ when compared to persons without migration background. 
Vaccination consultation and perceived information status. Receiving professional vaccination advice was independently associated with both, higher influenza and tetanus vaccine uptake (Tables 2 and 3). A total 56.0\% (95\%CI 55.2-56.9) of the interviewed persons reported to have received vaccination advice within the past five years. The most frequently reported source of vaccination advice was with 95.1\% (95\%CI 94.7-95.6) 'general practitioners'. Health insurance companies (reported by $2.5 \%$ of interviewed persons who received consultation) and official health authorities (1.6\%) played only a minor role as source of vaccination advice.

A total $87.9 \%(95 \% \mathrm{CI} 87.2-88.4)$ of respondents had at least one physician contact in the last 12 months, $10.6 \%$ (95\% CI 10.1-11.2) of respondents reported that their last physician contact was between 1 and 5 years ago, and 1.5\% (95\% CI 1.3-1.8) reported that it was longer than 5 years ago. Persons who had a physician contact in the last 12 months received significantly more often professional vaccination advice (58.1\% reported such an advice) than persons whose last physician contact was between 1 to 5 years ago $(42.9 \%$; $<<0.001)$ or more than 5 years ago $(30.0 \% ; \mathrm{p}<0.001)$.

Of all interviewed persons $79.3 \%(95 \%$ CI 78.6-80.0) felt sufficiently informed about the benefits of vaccination in general, and $62.2 \%$ (95\% CI 61.3.0-63.0) felt sufficiently informed about the risks of vaccination. Persons, who received professional vaccination advice, felt significantly more often sufficiently informed about the benefits $(91.9 \%$ vs. $63.2 \% ; \mathrm{p}<0.001)$ and risks $(74.1 \%$ vs. $46.8 \% ; \mathrm{p}<0.001)$ of vaccination than person without professional vaccination advice in the past 5 years.

Respondents with a two-sided migration background felt significantly less often sufficiently informed about the benefits and risks of vaccination ( $63.2 \%$ and $47.2 \%)$, and received significantly less often professional vaccination advice (45.2\%) when compared to persons with a one-sided or no migration background (for all $\mathrm{p}<0.001$ ). Similar observations were found for persons with a low socioeconomic status $(74.2 \%$ and $56.2 \%$; with $49.9 \%$ 
having received advice) when compared to persons with a medium or high socio-economic status (for all variables $\mathrm{p}<0.001$ ). 


\section{Discussion}

Due to the lack of an immunization registry in Germany, up-to-date country-level data on vaccination coverage in the general adult population and in specific vaccination target groups have to be assessed by surveys. ${ }^{7,16}$ With GEDA a large data source consisting of more than 21.000 interviews representative of the adult population in Germany is now available since 2009. It is the only data source for tetanus vaccination coverage in the adult population living in Germany for more than a decade. We were able to demonstrate that tetanus and seasonal influenza vaccination coverage in the target populations are unsatisfactorily low in Germany. Moreover, our study revealed a lack of knowledge in the population regarding information about the benefits and risks of vaccination, demonstrated the importance of private physicians as the main source of vaccination advice, and identified several potential barriers to tetanus and influenza vaccine uptake.

In accordance with other studies, ${ }^{17-19}$ receiving vaccination consultation was shown to be a strong enabling factor for both high influenza and tetanus vaccine uptake in our study. Persons who received a professional advice did not only feel better informed about the benefits but also about the risks of vaccination in general. Since only $56 \%$ of the respondents of this study received a vaccination advice in the past five years but $98 \%$ had visited a physician in this period, it is important to ensure that every contact with a physician is used to check vaccination status. Private physicians stood out as the main source of vaccination information and consultation for the general adult population. Therefore it is crucial to provide HCWs - especially private physicians - with information about vaccines and the respective vaccine-preventable diseases that is based upon current scientific evidence. ${ }^{20}$ Furthermore, HCWs should be enabled to adequately advice and inform patients regarding vaccination.

Differences in factors being associated with seasonal influenza and tetanus vaccine uptake have been identified. For influenza vaccination, higher coverage was identified in 
older people, in individuals with underlying chronic diseases, and in persons without perceived good health status. In contrast, higher tetanus vaccination coverage was associated with younger age, and not with perceived health status or chronic underlying diseases. These differences can be explained by the fact that seasonal influenza vaccination is recommended for specific risk groups, mainly older persons and persons with chronic underlying diseases.

The last nationwide assessment of tetanus vaccination coverage among adults was conducted in 1998 and revealed with 63\% a lower coverage as compared to our assessment $(73.1 \%) .{ }^{21}$ Current tetanus vaccination coverage in Germany is comparable to tetanus coverage in other European countries and the US. A study based on patient data collected in a Spanish medical emergency service in 2007 revealed among adults a tetanus vaccination coverage of $71.6 \%$ (95\% CI: $68.3-74.8) .{ }^{22}$ Based on data of the 2002 Health and Social Protection Survey, a tetanus vaccination coverage of $62.3 \%$ (95\%CI 61.1-63.5) was estimated for adults living in France. ${ }^{23}$ In the United States tetanus vaccine uptake was reported to be $61.6 \%$ (95\%CI: $60.6-62.5)$ in adults $\geq 18$ years based on data of the National Health Interview Survey $2008 .^{24}$ In all three surveys a sufficient tetanus vaccination status was defined as having received the last tetanus shot $\leq 10$ years ago. In our study we observed that tetanus vaccine uptake decreased with age. This observation was also made in other industrialized countries ${ }^{24,25}$ and is congruent to the fact that tetanus infections mainly occur among elderly persons in Germany. ${ }^{4}$ Thus, particularly older age-groups should be the target of individual tetanus vaccination catch-up activities.

Another important finding of our study was that persons with a SHI were significantly more likely to have a sufficient tetanus vaccination status than persons covered by PHI. The same observation was made for influenza vaccination among persons with an indication for this vaccination. A possible explanation for this finding, which remained stable in the multivariate analysis as an independent factor, might be that in Germany it is more complicated and time-consuming for someone with a PHI to receive the vaccination and to 
get the expenses reimbursed. Persons with PHI need to pay for the vaccine out-of-pocket, and reimbursement takes place not until the bills are send to the PHI. In addition, persons covered by SHI usually receive a vaccine dose from the physician's stock, whereas persons with PHI sometimes need a prescription from the physician first, then they need to go to the pharmacy to buy the vaccine, and finally they need to go back to the physician for the administration of the vaccine.

In our study, having a two-sided migration background was identified as a barrier to sufficient vaccination information and consultation as well as to tetanus vaccination. The latter observation is concordant with the findings of the 'German Health Interview and Examination Survey for Children and Adolescents' (KiGGS), which revealed significantly lower tetanus vaccination coverage (defined as a completed primary series of tetanuspreventing vaccinations) in adolescents aged 14-17 years when compared to those without migration background ( $\sim 89 \%$ vs. $\sim 97 \%)$. ${ }^{26}$ In the same survey insufficient vaccination coverage in adolescents (11-17 years) with migration background was detected for the vaccinations against Diphtheria, Haemophilus influenzae and Poliomyelitis, ${ }^{26}$ and in foreign born children (2-17 years) for the vaccination against measles. ${ }^{27}$ However, it should be noted that the sub-population 'persons with a two-sided migration background' is very heterogeneous. Therefore, the reasons for low vaccine uptake in this specific population are likely to be diverse.

Since STIKO-recommended vaccinations are free of charge in Germany, we did not expect that low socioeconomic status would be a barrier to basic vaccinations. Our results from multivariate analyses showed, however, that persons with a low socioeconomic status were significantly less often vaccinated against tetanus and received less frequently professional vaccination advice when compared to persons with a medium or high socioeconomic status. This was, however, different for influenza vaccine uptake, which was 7 percentage points higher in persons with low when compared to persons with high 
socioeconomic status (significant in bivariate but not multivariate analysis). Further research with focus on potential barriers to adult vaccination in different sub-groups of migrants as well as persons with low socioeconomic status is therefore necessary.

In our study significantly higher vaccination coverage was found in the eastern part of Germany for both vaccinations under investigation. Possible reasons for this observation have been discussed in detail in previous publications. ${ }^{8,9,14}$ In brief, mandatory vaccination practices in the former German Democratic Republic as well as differences in current implementation practices on the federal state level may contribute in general to a higher acceptance of vaccinations in the eastern federal states.

Our study has several limitations that need to be acknowledged. With $29.1 \%$ the response rate (Response Rate 3 as defined by $A A P O R^{15}$ ) was comparatively low. However, it should be mentioned that the chosen method of calculating a response rate is a very conservative approach and that the response rate in our study is comparable to other studies using this approach (e.g. CDC-Behavioral Risk Factor Surveillance System Report ${ }^{28}$ ). Considering the complex weighting procedures utilised in GEDA 2009 and the good cooperation rate $(51.2 \%)^{14}$ it can be assumed that data quality in terms of generalizability to the general adult population is overall good (compare Table 1). Since vaccination status was self-reported by the respondents it could not be corroborated by any medical records. However, several previous studies have found that self-report of recent influenza vaccination has an adequate degree of validity. ${ }^{29,30}$ It is also likely that accuracy of self-reporting for having received a seasonal influenza shot for the last season differs from that for having received tetanus vaccination within the past 10 years. The estimates for tetanus vaccination coverage might therefore have been more prone to recall bias than those for influenza vaccination. Finally, persons with insufficient knowledge of the German language or persons who could not be interviewed via telephone were excluded from our study. 


\section{Methods}

Study population and survey design. Details of the GEDA 2009 survey design have been described previously. ${ }^{14}$ In brief, GEDA 2009 is a national health telephone survey, representative of the adult population in Germany, which was conducted between July 2008 and June 2009. The study population $(n=21,262)$ included persons 18 years of age or older who were living in a private household in Germany and who were able to be contacted by landline telephone. Persons aged $\leq 17$ years and persons with an insufficient knowledge of the German language were excluded from the survey. The study protocol was approved by Germany's federal and regional data-protection commissioners. All data were collected and analyzed in an anonymous manner.

Response rates were calculated using Response Rate 3 as defined by the American Association for Public Opinion Research (AAPOR). ${ }^{15}$ Response Rate 3 is defined as the number of complete interviews divided by the number of interviews plus the number of noninterviews plus cases of unknown eligibility. Response Rate 3 uses an estimate for the proportion of cases of unknown eligibility that is potentially eligible. The cooperation rate at respondent level is defined as the proportion of all cases interviewed of all respondents ever contacted. This rate is calculated using only contacts with and refusals from known respondents. $^{15}$

Definition of variables. All participants were asked whether they were vaccinated against seasonal influenza during the last season (Appendix). Since survey respondents were contacted over a 12-month period, the 'last' influenza season is either season 2007/08 or 2008/09 - depending on point of time of interview. Univariate and multivariate analysis of factors potentially influencing influenza vaccine uptake was performed using the complete dataset without consideration of season. Influenza vaccination coverage was calculated for persons for whom influenza vaccination is recommended in Germany. In accordance with the 
STIKO 2008/09 recommendations ${ }^{1}$, we classified persons into the influenza vaccination target group if they reported (1) to be $\geq 60$ years of age, (2) to have underlying chronic diseases (chronic underlying respiratory, cardiovascular, liver or renal disease, cancer or diabetes), or (3) to work as a healthcare professional. Due to the study design, persons living in nursing or old people's homes, for which influenza vaccination is also recommended by STIKO, were included in the survey but not specifically asked if they live in such a home. Therefore, persons belonging to this target group were not identified and analyzed as a separate target group. Persons were regarded as vaccinated against tetanus if they reported to be vaccinated during the past 10 years. Persons were regarded as unvaccinated if they reported to have never received a tetanus vaccination or if the last tetanus shot was longer than 10 years ago. Persons were neither asked whether tetanus or influenza vaccination was offered to them nor if they actively declined a vaccination offer.

Socio-economic status levels were created as described by Lampert and Kroll on the basis of self-reported educational, income, and professional status of the interviewed person. ${ }^{32}$ The geographic region category 'Northwest/Middle' comprises the German federal states Schleswig-Holstein, Bremen, Lower Saxony, Hesse, Rhineland-Palatinate and Saarland (adult population: 20 million), 'Midwest' comprises North Rhine-Westphalia ( 15 million), 'East' comprises Mecklenburg-Vorpommern, Brandenburg, Berlin, Saxony-Anhalt, Thuringia and Saxony ( 14 million), and 'South' comprises Baden-Württemberg and Bavaria ( 19 million).

Interviewed persons were asked about the type of their health insurance (SHI, PHI, or other, which is defined as no health insurance, foreign health insurance, or unknown health insurance status) and potential migration background (none, one-sided when one parent and two-sided when both parents or the respondent were born abroad). Further questions included: Caring about personal health (yes/no); Self-assessment of general health status (good/not 
good); Smoking behaviour (current or past smoker/non-smoker); Alcohol consumption (regular/ never or occasional). Finally, interviewees were asked questions related to vaccination: Received professional vaccination advice within the last five years (yes/no); source of vaccination advice (general practitioner, health insurance company, official health authorities, other); feeling sufficiently informed about the benefits of vaccination in general / feeling sufficiently informed about potential harms of vaccination in general (yes/no). Respondents were not explicitly asked to review their medical records before responding.

Statistical analysis. Data were analysed using PASW version 18.0 for Windows (SPSS Inc., Chicago, USA). Univariate analyses were performed to determine the association between vaccination uptake and demographic, socio-economic, health-relevant and informational factors. A p-value $\leq 0.05$ was considered to indicate a statistically significant difference. Odds ratios (OR) and 95\% confidence intervals (CI) were calculated as appropriate. Variables potentially associated with vaccine uptake ( $\mathrm{p}$-value $<0.2$ in univariate analysis) were entered into a multivariate logistic regression model in a first step, followed by step-wise backward removal of variables with a $p$-value $>0.05$ to produce a final model. 
Conclusion. Despite an increase in tetanus and seasonal influenza vaccination coverage in German adults within the past ten years, ${ }^{11,21}$ our results indicate that tetanus vaccination coverage in the general adult population and influenza vaccination coverage in the target population is still unsatisfactorily low in Germany. Measures such as offering low-threshold vaccination services, implementing reminder systems for clients (potential vaccinees) and providers (physicians), and target group-oriented education strategies have been shown to improve vaccine uptake ${ }^{31}$ - especially when they are applied in combination - and should therefore be enhanced in Germany. As highlighted by the results of our study, private physicians should be a main target for enhanced activities related to vaccinations in Germany, since they are key figures in advising adults about vaccinations and informing them about the harms and benefits of the vaccines. In our study population, $88 \%$ had visited a physician in the past 12 months for any reason. Further research is necessary to evaluate communication channels for the efficient provision of vaccine-related information to private physicians and to assess their needs regarding vaccination-related information as well as patient recall and reminder systems. 


\section{Conflict of Interest}

The authors have declared no conflict of interests.

\section{Acknowledgements}

We would like to thank all colleagues at the Robert Koch Institute who were involved in the planning and implementation of the GEDA-Survey. 


\section{References}

1. Robert Koch-Institute. [Recommendations of the Standing Committee on Vaccination (STIKO)]. Epidemiologisches Bulletin 2010; 30:279-98.

2. German Medical Association. The Health Care System in Germany. 2011; [cited 20-06-2011; Available from: http://www.bundesaerztekammer.de/page.asp?his=4.3571].

3. German Federal Statistical Office. Diagnostic data of the hospitals by place of residence: Tetanus. 2011; [cited 06-07-2011; Available from: http://www.gbe-bund.de/].

4. Robert Koch-Institute. [Tetanus: Two Case-Reports]. Epidemiologisches Bulletin 2008; 24:192-5.

5. Robert Koch Institute. Tetanus: notification data. 2011; [cited 06-07-2011; Available from: http://www3.rki.de/SurvStat/].

6. Zucs P, Buchholz U, Haas W, Uphoff H. Influenza associated excess mortality in Germany, 1985-2001. Emerg Themes Epidemiol 2005; 2:6; PMID: 15969758; DOI: 10.1186/1742-7622-2-6.

7. Poggensee G, Reuss A, Reiter S, Siedler A. [Overview and assessment of available data sources to determine incidence of vaccine preventable diseases, vaccination coverage, and immune status in Germany]. Bundesgesundheitsbl 2009; 52:1019-28.

8. Wiese-Posselt M, Leitmeyer K, Hamouda O, Bocter N, Zollner I, Haas W, et al. Influenza vaccination coverage in adults belonging to defined target groups, Germany, 2003/2004. Vaccine 2006; 24:2560-6; PMID: 16414160; DOI:

10.1016/j.vaccine.2005.12.020.

9. Rehmet S, Ammon A, Pfaff G, Bocter N, Petersen LR. Cross-sectional study on influenza vaccination, Germany, 1999-2000. Emerg Infect Dis 2002; 8:1442-7; PMID: 12498661; DOI: 10.3201/eid0812.010497.

10. Blank PR, Freiburghaus AU, Ruf BR, Schwenkglenks MM, Szucs TD. Trends in influenza vaccination coverage rates in Germany over six seasons from 2001/02 to 2006/07. Med Klin (Munich) 2008; 103:761-8; DOI: 10.1007/s00063-008-1121-0 [doi].

11. Blank PR, Schwenkglenks M, Szucs TD. Disparities in influenza vaccination coverage rates by target group in five European countries: trends over seven consecutive seasons. Infection 2009; 37:390-400; PMID: 19768382; DOI: 10.1007/s15010-009-8467-y.

12. Reuss AM, Walter D, Feig M, Kappelmayer L, Buchholz U, Eckmanns T, et al. Influenza vaccination coverage in the 2004/05, 2005/06, and 2006/07 seasons: a secondary data analysis based on billing data of the German associations of statutory health insurance physicians. Dtsch Arztebl Int 2010; 107:845-50; DOI: 10.3238/arztebl.2010.0845 [doi]. 
13. German Federal Statistical Office. [Microcensus 2003 - Influenza vaccination since April 2002]. Wiesbaden, 2004. Available from: http://www.destatis.de.

14. Böhmer MM, Walter D, Müters S, Krause G, Wichmann O. Seasonal influenza vaccine uptake in Germany 2007/2008 and 2008/2009: Results from a national health update survey. Vaccine 2011; 29:4492-8; PMID: 21545822; DOI:

10.1016/j.vaccine.2011.04.039.

15. American Association for Public Opinion Research (AAPOR). Standard Definitions: Final Dipositions of Case Codes and Outcome Rates for Surveys. 2008; [cited 02-12-2010; Available from: http://www.aapor.org/AM/Template.cfm?Section=Standard_Definitions\&Template=/C M/ContentDisplay.cfm\&ContentID=1273].

16. Oppermann H, Wahl G, Borrmann M, Fleischer J. [Obligatory vaccination reporting in Saxony-Anhalt. Possibilities and limitations of establishing a computerized vaccination registry]. Bundesgesundheitsbl 2009; 52:1029-36; PMID: 19768390; DOI: 10.1007/s00103-009-0959-5.

17. Uddin M, Cherkowski GC, Liu G, Zhang J, Monto AS, Aiello AE. Demographic and socioeconomic determinants of influenza vaccination disparities among university students. J Epidemiol Community Health 2010; 64:808-13; PMID: 19828514; DOI: 10.1136/jech.2009.090852.

18. Bovier PA, Chamot E, Bouvier Gallacchi M, Loutan L. Importance of patients' perceptions and general practitioners' recommendations in understanding missed opportunities for immunisations in Swiss adults. Vaccine 2001; 19:4760-7; PMID: 11535327; DOI: 10.1016/S0264-410X(01)00223-7.

19. Burns VE, Ring C, Carroll D. Factors influencing influenza vaccination uptake in an elderly, community-based sample. Vaccine 2005; 23:3604-8; PMID: 15855020; DOI: 10.1016/j.vaccine.2004.12.031.

20. Baeyens JP. Ensuring the willingness to vaccinate and be vaccinated. Expert Rev Vaccines 2010; 9:11-4; PMID: 20192712; DOI: 10.1586/erv.10.28.

21. Reiter S, Rasch G. Immunizations. Federal Health Reporting (GBE) 2004; GBE Booklet 1:1-20;

22. del Corro MR, Vargas-Roman MI, Garcia RI, Prieto RG, de Miguel AG. Tetanus vaccination in adult population: coverage, registration and compliance. Hum Vaccin 2009; 5:98-104; PMID: 18758241; DOI: 10.4161/hv.5.2.6588.

23. Guthmann JP, Fonteneau L, Antona D, Lévy-Bruhl D. [Diphtheria, tetanus and poliomyelitis immunization coverage in French adults: results of the Health and Social Protection survey, 2002]. Bull Epidemiol Hebd 2007; 51-52:441-5. 
24. Centers for Disease Control and Prevention. Tetanus and pertussis vaccination coverage among adults aged $>/=18$ years --- United States, 1999 and 2008. MMWR Morb Mortal Wkly Rep 2010; 59:1302-6; PMID: 20948508.

25. Guthmann JP, Fonteneau L, Antona D, Levy-Bruhl D. [Factors associated with tetanus vaccination coverage in adults in France and with knowledge of vaccination status]. Med Mal Infect 2010; 40:560-7; PMID: 20400252; DOI:

10.1016/j.medmal.2010.03.009.

26. Poethko-Müller $\mathrm{C}$, Kuhnert R, Schlaud M. [Vaccination coverage and predictors for vaccination level. Results of the German Health Interview and Examination Survey for Children and Adolescents (KiGGS)]. Bundesgesundheitsblatt Gesundheitsforschung Gesundheitsschutz 2007; 50:851-62; PMID: 17514471; DOI: 10.1007/s00103-007-0248-0.

27. Poethko-Müller C, Ellert U, Kuhnert R, Neuhauser H, Schlaud M, Schenk L. Vaccination coverage against measles in German-born and foreign-born children and identification of unvaccinated subgroups in Germany. Vaccine 2009; 27:2563-9; PMID: 19428862; DOI: 10.1016/j.vaccine.2009.02.009.

28. Centers for Disease Control and Prevention. Behavioral Risk Factor Surveillance System: 2009 Summary Data Quality Report. 2010; [cited 08.09.2011; Available from: ftp://ftp.cdc.gov/pub/Data/Brfss/2009_Summary_Data_Quality_Report.pdf].

29. Mangtani P, Shah A, Roberts JA. Validation of influenza and pneumococcal vaccine status in adults based on self-report. Epidemiol Infect 2007; 135:139-43; PMID: 16740194; DOI: 10.1017/S0950268806006479.

30. Mac Donald R, Baken L, Nelson A, Nichol KL. Validation of self-report of influenza and pneumococcal vaccination status in elderly outpatients. Am J Prev Med 1999; 16:173-7; PMID: 10198654; DOI: 10.1016/S0749-3797(98)00159-7.

31. Ndiaye SM, Hopkins DP, Shefer AM, Hinman AR, Briss PA, Rodewald L, et al. Interventions to improve influenza, pneumococcal polysaccharide, and hepatitis $B$ vaccination coverage among high-risk adults: a systematic review. Am J Prev Med 2005; 28:248-79; PMID: 15894160; DOI: 10.1016/j.amepre.2005.02.016.

32. Lampert T, Kroll LE. [The measurement of socioeconomic status in socioepidemiological studies]. In: Richter M, Hurrelmann K, eds. Gesundheitliche Ungleichheit - Theorien, Konzepte und Methoden: VS Verlag für Sozialwissenschaften, 2006:297-319.

33. German Federal Statistical Office. Population. 2011; [cited 23-01-2011; Available from: https://www-genesis.destatis.de/genesis/online]. 


\section{Figure 1}

Tetanus and seasonal influenza vaccination coverage in the general population by age group, Germany 2008/09

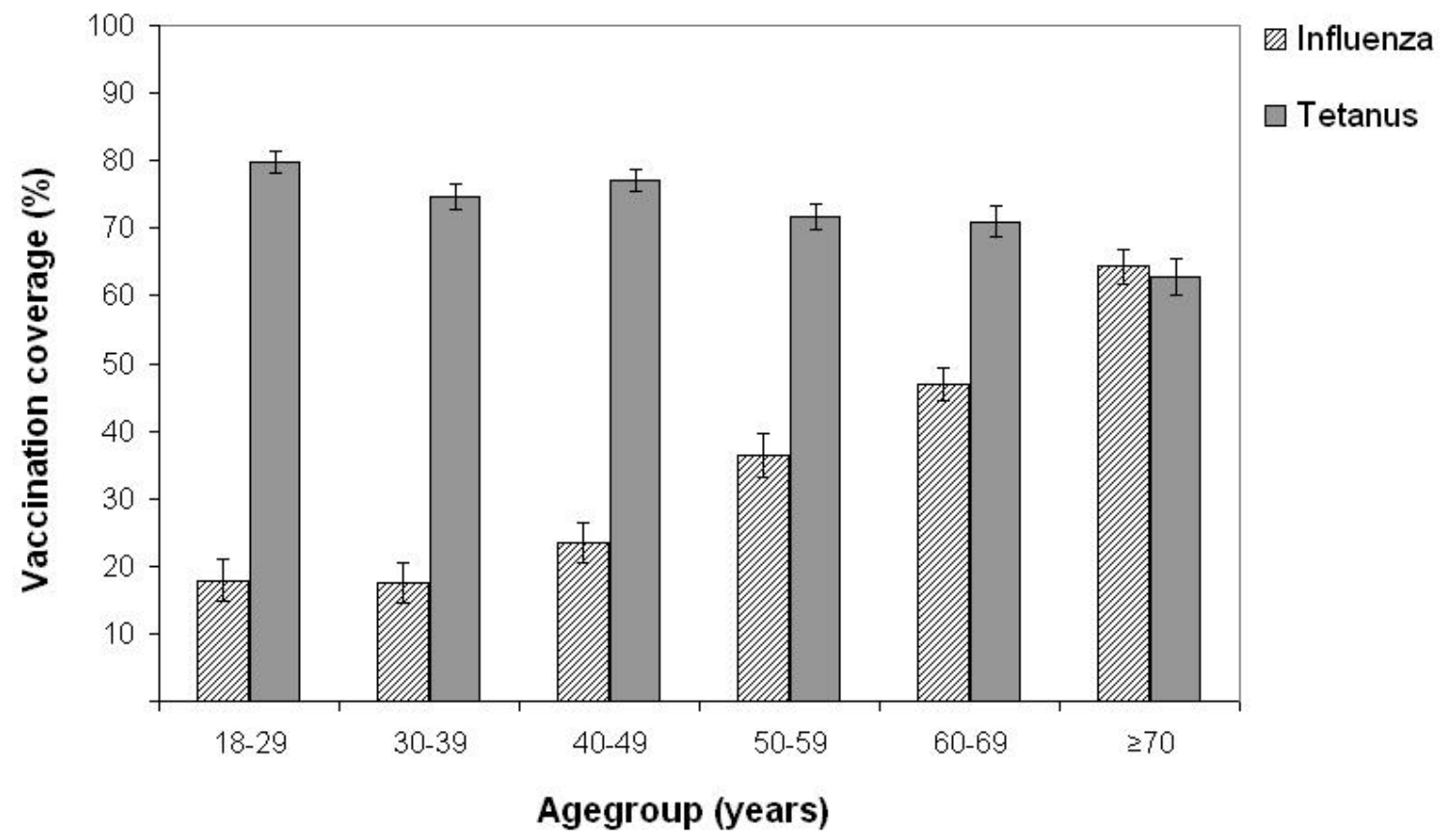


Tables

Table 1

Characteristics of persons aged $\geq 18$ years in the study sample and the general population, Germany, 2008/09

\begin{tabular}{|c|c|c|c|c|}
\hline \multirow[t]{2}{*}{ Characteristics } & \multicolumn{2}{|c|}{$\begin{array}{l}\text { Study population GEDA } \mathbf{0 9}^{*} \\
(n=21,262)\end{array}$} & \multicolumn{2}{|c|}{$\begin{array}{l}\text { General population, } \mathbf{2 0 0 8}^{* *} \\
(n=68,318,799)\end{array}$} \\
\hline & $n$ & $\%(95 \% \mathrm{CI})$ & $n$ & $\%$ \\
\hline \multicolumn{5}{|l|}{ Sex } \\
\hline Male & 10,310 & $48.5(47.6-49.4)$ & $33,165,264$ & 48.5 \\
\hline Female & 10,952 & $51.5(50.6-52.4)$ & $35,153,535$ & 51.5 \\
\hline \multicolumn{5}{|l|}{ Age } \\
\hline $18-39$ years & 7,021 & $33.0(32.2-33.8)$ & $22,135,423$ & 32.4 \\
\hline $40-59$ years & 7,774 & $36.6(35.7-37.4)$ & $25,225,632$ & 36,9 \\
\hline$\geq 60$ years & 6,467 & $30.4(29.5-31.3)$ & $20,957,744$ & 30.7 \\
\hline \multicolumn{5}{|l|}{ Geographic Region } \\
\hline Northwest/Middle & 6,263 & $29.5(28.7-30.3)$ & $20,126,061$ & 29.5 \\
\hline Midwest & 4,598 & $21.6(20.9-22.4)$ & $14,764,121$ & 21.6 \\
\hline East & 4,467 & $21.0(20.3-21.7)$ & $14,306,153$ & 20.9 \\
\hline South & 5,934 & $27.9(27.1-28.7)$ & $19,122,464$ & 28.0 \\
\hline \multicolumn{5}{|l|}{$\begin{array}{l}\text { Target group for influenza } \\
\text { vaccination }\end{array}$} \\
\hline Healthcare workers & 996 & $4.7(4.4-5.0)$ & $3,060,000$ & 4.5 \\
\hline $\begin{array}{l}\text { Persons with underlying } \\
\text { chronic conditions }\end{array}$ & 6,959 & $32.7(31.9-33.6)$ & unknown & \\
\hline Whole target population & 10,661 & $50.1(49.3-51.0)$ & unknown & \\
\hline
\end{tabular}

* weighted data; ${ }^{* *}$ data by the Federal Statistical Office of Germany ${ }^{33}$ 
Table 2

Univariate and multivariate analysis of factors potentially associated with tetanus vaccination coverage in the general population

\begin{tabular}{|c|c|c|c|}
\hline Variable & $\begin{array}{l}\text { Vaccination } \\
\text { coverage (\%) }\end{array}$ & $\begin{array}{l}\text { Univariate } \\
\text { OR }(95 \% \mathrm{CI})\end{array}$ & $\begin{array}{l}\text { Multivariate } \\
\text { OR (95\% CI) }\end{array}$ \\
\hline \multicolumn{4}{|l|}{ Sex } \\
\hline female & 71.6 & 1 (ref) & 1 (ref) \\
\hline male & 74.8 & $1.18(1.11-1.25)^{* *}$ & $1.20(1.11-1.29)^{* *}$ \\
\hline \multicolumn{4}{|l|}{ Age } \\
\hline $18-39$ & 77.3 & 1 (ref) & 1 (ref) \\
\hline $40-59$ & 74.8 & $0.87(0.80-0.94)^{* *}$ & $0.82(0.75-0.90)^{* *}$ \\
\hline$\geq 60$ & 66.7 & $0.59(0.54-0.63)^{* *}$ & $0.46(0.42-0.51)^{* *}$ \\
\hline \multicolumn{4}{|l|}{ Geographic Region } \\
\hline Northwest/Middle & 71.8 & 1 (ref) & 1 (ref) \\
\hline Midwest & 66.9 & $0.80(0.73-0.87)^{* *}$ & $0.90(0.81-0.99)^{*}$ \\
\hline East & 79.2 & $1.50(1.37-1.64)^{* *}$ & $1.37(1.23-1.52)^{* * *}$ \\
\hline South & 74.7 & $1.16(1.07-1.26)^{* *}$ & $1.07(0.97-1.17)$ \\
\hline \multicolumn{4}{|l|}{ Residency (population) } \\
\hline$<5,000$ & 77.6 & 1 (ref) & 1 (ref) \\
\hline $5,000-<20,000$ & 74.4 & $0.84(0.76-0.92)^{* *}$ & $0.93(0.84-1.04)$ \\
\hline $20,000-<100,000$ & 73.3 & $0.79(0.72-0.87)^{* *}$ & $0.84(0.76-0.93)^{*}$ \\
\hline$\geq 100,000$ & 69.4 & $0.66(0.60-0.72)^{* *}$ & $0.68(0.62-0.75)^{* *}$ \\
\hline \multicolumn{4}{|c|}{ Type of health insurance } \\
\hline PHI & 71.3 & 1 (ref) & 1 (ref) \\
\hline SHI & 73.2 & $1.10(1.00-1.21)$ & $1.26(1.12-1.41)^{* *}$ \\
\hline other & 77.4 & $1.37(1.11-1.69)^{*}$ & $1.24(0.97-1.60)$ \\
\hline \multicolumn{4}{|c|}{$\begin{array}{l}\text { Received vaccination advice within } \\
\text { past } 5 \text { years }\end{array}$} \\
\hline no & 58.2 & 1 (ref) & 1 (ref) \\
\hline yes & 84.8 & $4.00(3.74-4.28)^{* *}$ & $3.37(3.12-3.64)^{* *}$ \\
\hline \multicolumn{4}{|c|}{$\begin{array}{l}\text { Feel sufficiently informed about the } \\
\text { benefits of vaccination }\end{array}$} \\
\hline no & 58.6 & 1 (ref) & 1 (ref) \\
\hline yes & 77.2 & $2.39(2.22-2.56)^{* *}$ & $1.52(1.39-1.67)^{* *}$ \\
\hline \multicolumn{4}{|c|}{$\begin{array}{l}\text { Feel sufficiently informed about the } \\
\text { risks of vaccination }\end{array}$} \\
\hline no & 66.1 & 1 (ref) & NS \\
\hline yes & 77.4 & $1.76(1.65-1.87)^{* *}$ & \\
\hline \multicolumn{4}{|l|}{ Last physician contact } \\
\hline$<1$ year & 74.7 & 1 (ref) & 1 (ref) \\
\hline$\geq 1$ year $-<5$ years & 64.1 & $0.60(0.55-0.66)^{* *}$ & $0.68(0.61-0.76)^{* *}$ \\
\hline$\geq 5$ years & 45.4 & $0.28(0.23-0.35)^{* *}$ & $0.29(0.22-0.38)^{* * *}$ \\
\hline \multicolumn{4}{|l|}{ Socioeconomic Status } \\
\hline low & 67.4 & 1 (ref) & 1 (ref) \\
\hline medium & 74.1 & $1.39(1.29-1.50)^{* *}$ & $1.17(1.06-1.28)^{*}$ \\
\hline high & 76.0 & $1.54(1.39-1.69)^{* *}$ & $1.18(1.04-1.33)^{*}$ \\
\hline \multicolumn{4}{|l|}{ Migration background } \\
\hline none & 75.2 & 1 (ref) & 1 (ref) \\
\hline one-sided & 75.7 & $1.03(0.86-1.23)$ & $1.00(0.81-1.22)$ \\
\hline two-sided & 59.1 & $0.48(0.44-0.52)^{* *}$ & $0.60(0.53-0.67)^{* *}$ \\
\hline \multicolumn{4}{|c|}{ Caring about personal health } \\
\hline no & 70.5 & 1 (ref) & 1 (ref) \\
\hline yes & 75.4 & $1.28(1.20-1.36)^{* *}$ & $1.19(1.11-1.29)^{* *}$ \\
\hline \multicolumn{4}{|l|}{ Smoking } \\
\hline non-smoker & 73.9 & 1 (ref) & 1 (ref) \\
\hline current or past smoker & 72.5 & $0.93(0.88-0.99)^{*}$ & $0.91(0.85-0.99)^{*}$ \\
\hline \multicolumn{4}{|l|}{ Alcohol consumption } \\
\hline never/occasional & 72.2 & 1 (ref) & 1 (ref) \\
\hline regular & 75.7 & $1.20(1.12-1.29)^{* *}$ & $1.17(1.07-1.27)^{* *}$ \\
\hline \multicolumn{4}{|c|}{ Underlying chronic disease } \\
\hline no & 73.3 & 1 (ref) & NS \\
\hline yes & 72.9 & $0.98(0.92-1.04)$ & \\
\hline \multicolumn{4}{|c|}{ Perceived general health status } \\
\hline not good & 69.5 & 1 (ref) & NS \\
\hline good & 74.7 & $1.30(1.22-1.39)^{* *}$ & \\
\hline
\end{tabular}


Table 3

Univariate and multivariate analysis of factors potentially associated with influenza vaccination coverage in the recommended target population

\begin{tabular}{|c|c|c|c|}
\hline Variable & $\begin{array}{l}\text { Vaccination } \\
\text { coverage }(\%)\end{array}$ & $\begin{array}{l}\text { Univariate } \\
\text { OR }(95 \% \text { CI) }\end{array}$ & $\begin{array}{l}\text { Multivariate } \\
\text { OR (95\% CI) }\end{array}$ \\
\hline \multicolumn{4}{|l|}{ Sex } \\
\hline female & 44.5 & 1 (ref) & \multirow[t]{2}{*}{ NS } \\
\hline male & 43.5 & $0.96(0.89-1.04)$ & \\
\hline \multicolumn{4}{|l|}{ Age } \\
\hline $18-39$ & 17.5 & 1 (ref) & $1(\mathrm{ref})$ \\
\hline $40-59$ & 30.0 & $2.02(1.73-2.35)^{* * *}$ & $2.02(1.71-2.38)^{* *}$ \\
\hline$\geq 60$ & 56.3 & $6.07(5.29-6.95)^{* *}$ & $6.55(5.64-7.61)^{* *}$ \\
\hline \multicolumn{4}{|l|}{ Geographic Region } \\
\hline Northwest/Middle & 40.2 & 1 (ref) & $1(\mathrm{ref})$ \\
\hline Midwest & 43.1 & $1.13(1.01-1.25)^{*}$ & $1.20(1.06-1.36)^{*}$ \\
\hline East & 58.9 & $2.13(1.91-2.38)^{* *}$ & $2.00(1.76-2.27)^{* *}$ \\
\hline South & 37.9 & $0.91(0.82-1.01)$ & $0.90(0.80-1.01)$ \\
\hline \multicolumn{4}{|l|}{ Residency (population) } \\
\hline$<5,000$ & 45.1 & 1 (ref) & \multirow[t]{4}{*}{ NS } \\
\hline $5,000-<20,000$ & 43.5 & $0.94(0.84-1.05)$ & \\
\hline $20,000-<100,000$ & 43.7 & $0.95(0.85-1.06)$ & \\
\hline$\geq 100,000$ & 44.0 & $0.96(0.86-1.07)$ & \\
\hline \multicolumn{4}{|c|}{ Type of health insurance } \\
\hline PHI & 37.2 & 1 (ref) & 1 (ref) \\
\hline SHI & 45.1 & $1.39(1.22-1.58)^{* *}$ & $1.32(1.14-1.53)^{* *}$ \\
\hline other & 31.8 & $0.79(0.57-1.08)$ & $1.03(0.72-1.47)$ \\
\hline \multicolumn{4}{|c|}{$\begin{array}{l}\text { Received vaccination advice within } \\
\text { past } 5 \text { years }\end{array}$} \\
\hline no & 29.3 & 1 (ref) & 1 (ref) \\
\hline yes & 54.7 & $2.90(2.67-3.15)^{* *}$ & $3.05(2.77-3.35)^{* *}$ \\
\hline \multicolumn{4}{|c|}{$\begin{array}{l}\text { Feel sufficiently informed about the } \\
\text { benefits of vaccination }\end{array}$} \\
\hline no & 29.9 & 1 (ref) & \multirow[t]{2}{*}{ NS } \\
\hline yes & 47.1 & $2.09(1.88-2.34)^{* *}$ & \\
\hline \multicolumn{4}{|c|}{$\begin{array}{l}\text { Feel sufficiently informed about the } \\
\text { risks of vaccination }\end{array}$} \\
\hline no & 36.7 & 1 (ref) & 1 (ref) \\
\hline yes & 47.5 & $1.56(1.43-1.69)^{* *}$ & $1.16(1.05-1.29)^{*}$ \\
\hline \multicolumn{4}{|l|}{ Last physician contact } \\
\hline$<1$ year & 46.4 & 1 (ref) & 1 (ref) \\
\hline$\geq 1$ year $-<5$ years & 19.6 & $0.28(0.24-0.34)^{* *}$ & $0.37(0.30-0.44)^{* *}$ \\
\hline$\geq 5$ years & 26.7 & $0.42(0.28-0.63)^{* *}$ & $0.44(0.27-0.70)^{*}$ \\
\hline \multicolumn{4}{|l|}{ Socioeconomic Status } \\
\hline low & 47.7 & 1 (ref) & \multirow[t]{3}{*}{ NS } \\
\hline medium & 43.7 & $0.85(0.78-0.93)^{*}$ & \\
\hline high & 40.3 & $0.74(0.66-0.84)^{* *}$ & \\
\hline \multicolumn{4}{|l|}{ Migration background } \\
\hline none & 45.0 & 1 (ref) & 1 (ref) \\
\hline one-sided & 44.0 & $0.96(0.78-1.19)$ & $1.00(0.78-1.28)$ \\
\hline two-sided & 35.9 & $0.69(0.60-0.78)^{* *}$ & $1.23(1.05-1.44)^{*}$ \\
\hline \multicolumn{4}{|c|}{ Caring about personal health } \\
\hline no & 38.0 & 1 (ref) & 1 (ref) \\
\hline yes & 48.4 & $1.53(1.42-1.66)^{* *}$ & $1.26(1.15-1.38)^{* *}$ \\
\hline \multicolumn{4}{|l|}{ Smoking } \\
\hline non-smoker & 48.6 & 1 (ref) & 1 (ref) \\
\hline current or past smoker & 40.1 & $0.71(0.66-0.76)^{* *}$ & $0.91(0.83-1.00)^{*}$ \\
\hline \multicolumn{4}{|l|}{ Alcohol consumption } \\
\hline never/occasional & 44.2 & 1 (ref) & NS \\
\hline regular & 43.2 & $0.96(0.88-1.05)$ & \\
\hline Underlying chronic di & & & \\
\hline no & 35.9 & 1 (ref) & 1 (ref) \\
\hline yes & 50.9 & $1.85(1.71-2.00)^{* *}$ & $1.37(1.24-1.51)^{* *}$ \\
\hline Perceived general heal & & & \\
\hline not good & 52.7 & 1 (ref) & 1 (ref) \\
\hline good & 37.5 & $0.54(0.50-0.58)^{* *}$ & $0.72(0.65-0.79)^{* *}$ \\
\hline
\end{tabular}

